\title{
On the Existence of Positive Solution for nth Order Differential Equation for Boundary Value Problems
}

\author{
Mohamed Seddeek ${ }^{1}$, Sayeda Nabhan Odda ${ }^{2}$ \\ ${ }^{1}$ Department of Mathematics, Faculty of Science, Helwan University, Cairo, Egypt \\ ${ }^{2}$ Department of Mathematics, Faculty of Women, Ain Shams University, Cairo, Egypt \\ Email address: \\ seddeek_m@hotmail.com (M. Seddeek)
}

\section{To cite this article:}

Mohamed Seddeek, Sayeda Nabhan Odda. On the Existence of Positive Solution for nth Order Differential Equation for Boundary Value Problems. Mathematics and Computer Science. Vol. 2, No. 4, 2017, pp. 47-50. doi: 10.11648/j.mcs.20170204.13

Received: May 11, 2017; Accepted: June 6, 2017; Published: July 31, 2017

\begin{abstract}
We considering the problem of solving a nonlinear differential equation in the Banach space of real functions and continuous on a bounded and closed interval. By means of the fixed point theory for a strict set contraction operator, this paper investigates the existence, nonexistence, and multiplicity of positive solutions for a nonlinear higher order boundary value problem.
\end{abstract}

Keywords: Positive Solutions, Fixed-Point Theorem, Operator Equations, Banach Space

\section{Introduction}

In the current paper, we considering the problem of solving a nonlinear differential equation of nth order. We will try to find the solutions of this equation in the Banach space. The main tool used in our investigations for existence of positive solutions for the nonlinear nth order boundary value problem. Let us mention that the theory of nonlinear differential equations has many useful applications in describing numerous events and problems of the real world. On the other hand, the existence results of positive solutions for integer order differential equations have been studied by several researchers (see [6-9] and the references therein), but, as far as we know, only a few papers consider the BVP for higher order nonlinear differential equations in Banach space of real functions and continuous on a bounded and closed interval, (see [1, 3, 5], and the references therein), So, the aim of this paper is to fill this gap. In this paper, we will obtain the existence and nonexistence of positive solution for the BVP (1), (2) and (3) in Banach space. The results presented in this paper seems to be new and original. The generalize equations are often applicable in engineering, mathematical physics, economies, and biology.

\section{Notation, Definition, and Auxiliary Results}

Theorem $2.1[1,2,9]$.

Assume that $\mathrm{U}$ is a relatively open subset of convex set $\mathrm{K}$ in Banach space E. Let $N: \bar{U} \rightarrow K$ be a compact map with $o \in U$. Then either

(i) $\mathrm{N}$ has a fixed point in $\bar{U}$; or

(ii) There is a $u \in U$ and a $\lambda \in(0,1)$ such that $u=\lambda N u$.

Definition 2.1 An operator is called completely continuous if it is continuous and maps bounded sets into precompacts.

Definition 2.2 Let $\mathrm{E}$ be a real Banach space. A nonempty closed convex set $K \subset E$ is called cone of $E$ if it satisfies the following conditions:

(i) $x \in K, \sigma \geq o$ implies $\sigma x \in K$;

(ii) $x \in K,-x \in K$ implies $x=o$.

\section{Main Result}

In this section, we will study the existence and nonexistence of positive solutions for the nonlinear boundary value problem:

$$
\begin{gathered}
u^{(n)}(t)=f(t, u(t)), \quad 0<\mathrm{t}<1, \\
u^{\prime}(1)=u^{\prime \prime}(0)=u^{\prime \prime \prime}(0)=u^{(4)}(0)=\cdots=u^{n-1}(0)=0, \text { for all }
\end{gathered}
$$




$$
n \geq 2
$$

$\alpha u(0)+\beta u^{\prime}(0)=0, \quad$ where $\alpha, \beta \geq 0, \quad \alpha+\beta \succ 0$

This is equivelent for an integral equation:

$$
\begin{gathered}
u(t)=\int_{0}^{1} G(t, s) f(s, u(s)) d s, \\
\begin{array}{c}
\text { Theorem 3.1. Under conditions } \\
\text { has a unique solution. } \\
\text { Proof. Applying the Laplace tran } \\
\text { get }
\end{array} \\
s^{n} \bar{u}(s)-s^{n-1} u(0)-s^{n-2} u^{\prime}(0)-s^{n-3} u^{\prime \prime}(0)-\cdots-s u^{n-2}(0)-u^{n-1}(0)=\bar{y}(s) \\
s^{n} \bar{u}(s)-s^{n-1} A+s^{n-2} \frac{\alpha}{\beta} A-s^{n-3} u^{\prime \prime}(0)-\cdots-u^{n-1}(0)=\bar{y}(s)
\end{gathered}
$$

Theorem 3.1. Under conditions (2) and (3), equation (1)

Proof. Applying the Laplace transform to equation (1) we

Where $\bar{u}(s)$ and $\overline{\mathrm{y}}(\mathrm{s})$ is the Laplace transform of $u(t)$ and $\mathrm{y}(\mathrm{t})$ respectively. The laplace inversion of Eq.(3.4) gives the final solution as:

$$
\begin{gathered}
u(t)=\frac{\beta}{\alpha} \int_{0}^{1} \frac{(1-s)^{n-2}}{(n-2) !} f(s, u(s)) d s-\int_{0}^{1} t \frac{(1-s)^{n-2}}{(n-2) !} f(s, u(s)) d s \\
+\int_{0}^{t} \frac{(t-s)^{n-1}}{(n-1) !} f(s, u(s)) d s
\end{gathered}
$$

The proof is complete.

Defining $T: X \rightarrow X$ as:

$$
\begin{gathered}
T u(t)=\frac{\beta}{\alpha} \int_{0}^{1} \frac{(1-s)^{n-2}}{(n-2) !} f(s, u(s)) d s-\int_{0}^{1} t \frac{(1-s)^{n-2}}{(n-2) !} f(s, u(s)) d s \\
+\int_{0}^{t} \frac{(t-s)^{n-1}}{(n-1) !} f(s, u(s)) d s
\end{gathered}
$$

super norm. We have the following result for operator $\mathrm{T}$.

Lemma 3.1

Assume that $f:[0,1] \times R \rightarrow R$ is continuous function, then $\mathrm{T}$ is completely continuous operator.

Proof: It is easy to see that $\mathrm{T}$ is continuous. For $u \in M=\{u \in X ;\|u\| \leq l, l \succ 0\}$, we

Where $\mathrm{X}=\mathrm{C}[0,1]$ is the Banach space endowed with the

$$
\begin{gathered}
|T u(t)|=\left|\begin{array}{c}
\frac{\beta}{\alpha} \int_{0}^{1} \frac{(1-s)^{n-2}}{(n-2) !} f(s, u(s)) d s-\int_{0}^{1} t \frac{(1-s)^{n-2}}{(n-2) !} f(s, u(s)) d s \mid \\
+\int_{0}^{t} \frac{(t-s)^{n-1}}{(n-1) !} f(s, u(s)) d s
\end{array}\right| \\
\leq \frac{\beta}{\alpha} \int_{0}^{1} \frac{(1-s)^{n-2}}{(n-2) !}|f(s, u(s))| d s+\int_{0}^{1} t \frac{(1-s)^{n-2}}{(n-2) !}|f(s, u(s))| d s+\int_{0}^{t} \frac{(t-s)^{n-1}}{(n-1) !}|f(s, u(s))| d s \quad \leq \frac{\beta}{\alpha} \frac{L}{(n-1) !}+\mathrm{t} \frac{L}{(n-1) !}+t^{n} \frac{\mathrm{L}}{\mathrm{n} !}
\end{gathered}
$$

where $L=\max _{0 \leq t \leq 1,|u| \leq 1}|f(t, u(t))|+1$,

so $\mathrm{T}(\mathrm{M})$ is bounded. Next we shall show the equicontinuity of $\overline{T(M)} . \forall \varepsilon \succ 0, t_{1} \prec t_{2} \in[0,1]$.

Let $, \eta \prec\left\{\frac{(n-1) ! \varepsilon}{2 L}\right\}, \quad \gamma \prec\left\{\frac{\varepsilon n !}{2 L}\right\}, \quad$, we have

$$
\mathrm{t}_{2}-\mathrm{t}_{1} \prec \eta, \quad \mathrm{t}_{2}^{\mathrm{n}}+\mathrm{t}_{1}^{\mathrm{n}} \prec \gamma
$$

$$
\begin{aligned}
\left|T u\left(t_{2}\right)-T u\left(t_{1}\right)\right|=\left|\begin{array}{l}
-\int_{0}^{1} \frac{(1-s)^{n-2}}{(n-2) !}\left(t_{2}-t_{1}\right) f(s, u(s)) d s \\
+\int_{0}^{t_{2}} \frac{\left(t_{2}-s\right)^{n-1}}{(n-1) !} f(s, u(s)) d s-\int_{0}^{t_{1}} \frac{\left(t_{1}-s\right)^{n-1}}{(n-1) !} f(s, u(s)) d s
\end{array}\right| \\
\qquad L \frac{\left(t_{2}-t_{1}\right)}{(n-1) !}+\frac{L t_{2}{ }^{n}}{n !}+\frac{\mathrm{Lt}_{1}{ }^{n}}{\mathrm{n} !}
\end{aligned}
$$




$$
\begin{aligned}
& \leq \frac{\mathrm{L}}{(\mathrm{n}-1) !} \eta+\frac{L \gamma}{n !} \\
& \leq \frac{\varepsilon}{2}+\frac{\varepsilon}{2}
\end{aligned}
$$

Thus $\overline{T(M)}$ is equicontinuous. The Arzela-Ascoli theorem implies that the operator $\mathrm{T}$ is completey continuous.

Theorem 3.2

Assume that $f:[0,1] x R \rightarrow R$ is continuous function, and there exist constants $|f(t, u(t))| \leq c_{1}|u|+c_{2}$ for all $t \in[0,1]$.

Then the boundary value problem (1), (2) and (3) has a solution.

Proof: Following [2, 4 and 10], we will apply the nonlinear alternative theorem to prove that $\mathrm{T}$ has one fixed point.

Let $\Omega=\{u \in X ;\|u\|<R\}$, be open subset of $X$, where $R \succ \max \left(6\left\{\frac{\beta}{(n-1) ! \alpha} c_{1}, \frac{1}{(n-1) !} c_{1}, \frac{c_{1}}{n !}, \frac{\beta}{(n-1) ! \alpha} c_{2}, \frac{1}{(n-1) !} c_{2}, \frac{c_{2}}{n !}\right\}\right)$ We suppose that there is a point $u \in \partial \Omega$ and $c_{1} \in(0,1)$ such that $u=T u$. So, for $u \in \partial \Omega$, we have: $0 \prec \mathrm{c}_{1} \prec \min \left(\frac{(n-1) ! \alpha}{\beta}, \quad(\mathrm{n}-1) !, n !\right), \quad \mathrm{c}_{2} \succ o$, such that

$$
\begin{gathered}
|T u(t)|=\left|\begin{array}{rl}
\frac{\beta}{\alpha} \int_{0}^{1} \frac{(1-s)^{n-2}}{(n-2) !} f(s, u(s)) d s-\int_{0}^{1} \frac{(1-s)^{n-2}}{(n-2) !} t f(s, u(s)) d s \mid & +\int_{0}^{t} \frac{(t-s)^{n-1}}{(n-1) !} f(s, u(s)) d s
\end{array}\right| \\
\leq \frac{\beta}{\alpha} \int_{0}^{1} \frac{(1-s)^{n-2}}{(n-2) !}|f(s, u(s))| d s+\int_{0}^{1} \frac{(1-s)^{n-2}}{(n-2) !} t|f(s, u(s))| d s+\int_{0}^{t} \frac{(t-s)^{n-1}}{(n-1) !}|f(s, u(s))| d s \\
\leq \frac{\beta}{\alpha} \int_{0}^{1} \frac{(1-s)^{n-2}}{(n-2) !}\left(c_{1}|u(s)|+c_{2}\right) d s+\int_{0}^{1} \frac{(1-s)^{n-2}}{(n-2) !} t\left(c_{1}|u(s)|+c_{2}\right) d s+\int_{0}^{t} \frac{(t-s)^{n-1}}{(n-1) !}\left(c_{1}|u(s)|+c_{2}\right) d s \\
\leq \frac{\beta}{(n-1) ! \alpha}\left(c_{1}|u(s)|\right)+\frac{1}{(n-1) !}\left(c_{1}|u(s)|\right)+\frac{1}{n !}\left(c_{1}|u(s)|\right)+ \\
\quad+\frac{R}{6}+\frac{R}{6}+\frac{R}{6}+\frac{R}{6}+\frac{R}{6}+\frac{R}{6}=\mathrm{R},
\end{gathered}
$$

which implies that $\|T\| \neq R=\|u\|$, that is a contraction. Then the nonlinear alternative theorem implies that $\mathrm{T}$ has a fixed point $u \in \bar{\Omega}$, that is, problem(1), (2) and (3) has a solution $u \in \bar{\Omega}$.

Finally, we give an example to illustrate the results obtained in this paper.

Example: For the boundary value problem (1), (2), and (3) we solve:

$$
u^{7}(t)=\frac{7 u+1}{u^{3}+1}
$$

Apply the theorem 3.2 with $\alpha=1$ and $\beta=1$. Then we have $c_{1} \prec \min \left(\frac{6 ! \alpha}{\beta}, 6 !, 7 !\right)$. We conclude that the problem (7) has a solution.

\section{Conclusion}

In This Paper we investigated the existence, nonexistence, and multiplicity of positive solutions for a nonlinear higher order boundary value problem on a bounded and closed interval by means of the fixed point theory for a strict set contraction operator. Let us mention that the theory of nonlinear differential equations has many useful applications in describing numerous events and problems of the real world. The results presented in this paper seem to be new and original. They generalize equations are often applicable in engineering, mathematical physics, economies, and biology.

\section{References}

[1] Guo, D and Lakshmikantham, V., 1988, Nonlinear Problems in Abstract Cones, Academic Press, San Diego.

[2] R. P. Agarwal, M. Meehan and D. O'Regan, Fixed Point and Applications, Cambridge University Press, Cambridge, 2001.

[3] Sun, H. and Wen, W., 2006, On the Number of positive solutios for a nonlinear third order boundary value problem, International Journal of Difference Equations, 1, 165- 176. 
[4] J. R. L. Webb, Positive solutions for some higher order nonlocal boundary value problems, Electronic journal of qualitative theory of differential equations spec. Ed. I, 2009 No.1, 1-14.

[5] F. Minhos and A. I. Santos, Higher order two-point boundary value problems with asym8] Qi Wang, Yanping Guo, and Yude Ji, Positive solutions for fourth-order nonlinear differential equation with integral boundary conditions, Discrete Dynamics in Nature and Society Volume 2013, (2013) 1-10.

[6] Yongping Sun and Xiaoping Zhang, Existence and nonexistence of positive solutions for fractional-order twopoint boundary value problems, Advances in Difference Equations, Volume 2013 (2014) 1-11.
[7] S. N. Odda "On the existence of positive solution for the classe of nth order nonlinear differential equations" Journal of Advances in Mathematics, Vol. 9, No. 2 (2014) 1755-1759.

[8] S. N. Odda "On the existence of positive solution for the classe of nth order nonlinear differential equations" Journal of Advances in Mathematics, Vol. 9, No. 2 (2014) 1755-1759.

[9] S. N. Odda "Positive solutions of a singular 4th order twopoint boundary value problem " Journal of Natural Sciences and Mathematics, Vo 9, No.2 (2017).

[10] S. N. Odda "On The Existence Positive Solution For 5th Order Differential Equation For Boundary Value Problems "Journal of Natural Sciences and Mathematics, Vo 10, No.1 (2017). 